\title{
Avaliação da prevalência das seis chaves de oclusão de Andrews, em jovens brasileiros com oclusão normal natural
}

\author{
Liliana Ávila Maltagliati*, Luciana Andrade do Prado Montes**, Fernanda Marcondes Machado Bastia**,

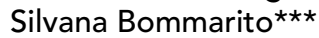

Resumo

Objetivos: diante da larga utilização e difusão dos conceitos das seis chaves de oclusão de Andrews como instrumento de diagnóstico e planificação de tratamento, realizamos este trabalho com o objetivo de avaliar a prevalência destas características numa amostra de oclusão normal brasileira. Metodologia: 61 modelos de estudo de indivíduos brasileiros, leucodermas e com oclusão normal natural foram estudados. Foi observada a freqüência com que as seis chaves foram encontradas em cada indivíduo, bem como quais as chaves de maior e menor freqüência. Resultados e Conclusões: os resultados demonstraram que a grande maioria dos indivíduos apresentou de uma a três chaves de oclusão e nenhum dos modelos apresentou as seis chaves de oclusão. As características observadas com maior freqüência foram a presença da curva de Spee suave (100\%), contatos interproximais justos $(42,6 \%)$ e inclinações corretas $(34,4 \%)$. A baixa prevalência de oclusões normais naturais na população, que preencham os requisitos propostos por Andrews, leva-nos a refletir sobre a busca desenfreada por padrões tão rígidos.

Palavras-chave: Ortodontia. Oclusão dentária. Oclusão normal.

\section{INTRODUÇÃO}

Alguns autores propuseram classificações para as más oclusões, buscando agrupar os indivíduos com características semelhantes em diferentes classes ou padrões. O método classificatório tem como principal objetivo estabelecer parâmetros para um bom diagnóstico, com conseqüente planificação eficaz do tratamento e avaliação dos resultados obtidos. Além disso, possibilita a criação de uma linguagem ortodôntica universal que facilita sobremaneira a troca de informações entre os pro- fissionais e a comparação entre os casos.

A primeira classificação das más posições dentárias foi publicada por Fox (1803 apud WEINBERGER $^{7}, 1926$ ) e se baseou nas inclinações dos incisivos superiores dividindo o mau posicionamento dentário em quatro grupos: 1) inclinação lingual de apenas um dos incisivos centrais, enquanto o outro está bem posicionado; 2) inclinação dos dois incisivos centrais para lingual, posicionando-se por trás dos inferiores; 3) inclinação correta dos incisivos centrais, porém

* Professora Titular da UMESP. Coordenadora do Curso de Especialização em Ortodontia da UMESP.

** Mestranda do Programa de Pós-graduação de Mestrado em Odontologia. Área de Concentração em Ortodontia.

*** Professora Titular da UMESP. 
com os laterais lingualizados, localizando-se por trás dos inferiores, no momento da intercuspidação, e 4) inclinação dos quatro incisivos superiores para lingual, ocasionando mordida cruzada anterior.

Delabarre (1819 apud WEINBERGER $\left.{ }^{7}, 1926\right)$ classificou as alterações esqueléticas em cinco grupos: protrusão maxilar, retrusão mandibular, prognatismo mandibular, mordida aberta esquelética e mordida cruzada posterior.

O sistema de classificação das más oclusões, desenvolvido por Angle $^{3}$ em 1899, tornou-se o mais conhecido e utilizado no mundo até a atualidade, provavelmente pela simplicidade de compreensão e abrangência. Em sua publicação o autor já mencionava que, para um diagnóstico ortodôntico correto, seria necessária a clara compreensão do que é uma oclusão dentária normal ou ideal e descreveu os aspectos dentários que considerava representativos desta oclusão. Citou que, quando em oclusão, cada arco dentário descreve uma curva graciosa e os dentes estão posicionados em harmonia com seus adjacentes e com os dentes do arco oposto. $\mathrm{O}$ arco inferior deveria ser um pouco menor que o superior de forma que, em oclusão, as superfícies vestíbulo-linguais dos dentes superiores projetariam-se levemente sobre as dos inferiores e a chave de oclusão estaria localizada na altura dos primeiros molares permanentes. Assim, a relação correta seria aquela em que a cúspide mésio-vestibular do primeiro molar superior estaria posicionada no sulco mésio-vestibular do molar inferior. Baseando-se no posicionamento desses dentes, classificou as más oclusões em Classes I, II e III com suas divisões e subdivisões. Em 1907, Angle ${ }^{4}$ redefiniu a classificação e modificou o conceito anteriormente descrito, salientando também a participação dos maxilares na determinação das más oclusões.

Com o surgimento do cefalostato ${ }^{6}$, em 1931, foi possivel estudar o crescimento facial $^{8}$ por meio de telerradiografias obtidas de forma padronizada. O diagnóstico, que outrora fora embasado principalmente no estudo dos modelos, a partir de então, passou a contar com novas informações provenientes das análises cefalométricas, fornecendo maiores subsídios para o planejamento da terapia ortodôntica.

Só em 1972, Andrews ${ }^{2}$ realizou a pesquisa que identificou "As seis chaves da oclusão normal", descrevendo as características fundamentais de uma oclusão dentária sob o ponto de vista morfológico, servindo também como guia para a finalização adequada dos tratamentos ortodônticos. Em sua pesquisa com modelos ortodônticos de indivíduos com oclusão normal, Andrews ${ }^{2}$ reconheceu e estabeleceu seis características comuns entre estes modelos: 1) Relação dos molares - os primeiros molares permanentes superiores devem mostrar três pontos de contato evidentes com os dentes antagonistas; a superfície distal da crista marginal do primeiro molar permanente superior contacta e oclui com a superfície mesial da crista marginal mesial do segundo molar permanente inferior; a cúspide mésiovestibular do primeiro molar permanente superior oclui dentro do sulco existente entre a cúspide mésio-vestibular e a mediana do primeiro molar inferior e a cúspide mésio-palatina do primeiro molar permanente superior adapta-se à fossa central do primeiro molar permanente inferior; 2) Angulação das coroas - a porção cervical do longo eixo de cada coroa encontra-se distalmente à sua porção oclusal; 3) Inclinação das coroas - a porção cervical do longo eixo da coroa dos incisivos superiores encontra-se por lingual à superfície incisal, aumentando a inclinação lingual progressivamente na região posterior; 4) Rotações - não deve haver rotações dentárias indesejáveis; 5) Contatos interproximais - não deve haver espaços interproximais; 6) Curva de Spee - deve apresentar-se plana ou suave. $\mathrm{O}$ autor relatou que as chaves eram interdependentes de um sistema estrutural e que serviam como base para a avaliação dos pacientes ortodônticos, sendo que a falha de uma ou mais chaves, indicaria uma oclusão inadequada.

A partir dos resultados obtidos, Andrews desenvolveu o sistema de braquetes pré-ajustados, no qual as características de normalidade de cada dente foram incorporadas aos acessórios ortodônticos, 
facilitando sobremaneira o tratamento e a confecção dos arcos. Desde então, não surgiram mais propostas para classificação de modelos com oclusão normal, apenas para modelos com más oclusões, como por exemplo, as classificações propostas por Moyers $^{8}$ e Woodside ${ }^{9}$.

Considerando que os valores normativos dentários e cefalométricos, propostos pelos diversos autores, estão baseados em pesquisas realizadas com amostras obtidas a partir de grupos raciais e étnicos com características peculiares e que, portanto, podem não ser aplicáveis em outras populações, Brangeli ${ }^{5}$ realizou um estudo para avaliar a prevalência das seis chaves de oclusão de Andrews numa amostra de brasileiros, leucodermas, com oclusão normal natural, selecionados de acordo com os critérios propostos por Angle $\mathrm{e}^{3}$, o que denominou de oclusão normal. Os resultados deste estudo revelaram que nenhum caso da amostra apresentou todas as seis chaves de oclusão; apenas $10 \%$ demonstrou possuir cinco chaves, enquanto $15 \%, 23 \%$ e $34 \%$ dos casos da amostra apresentaram, respectivamente, quatro, três e duas chaves. Por outro lado, seis casos não apresentaram nem ao menos uma das chaves de modo satisfatório e $11 \%$ preencheram satisfatoriamente os requisitos de apenas uma chave.

Seguindo a mesma linha de pesquisa do trabalho realizado em $2001^{5}$, propusemo-nos no trabalho atual a verificar também qual a prevalência das chaves de oclusão de Andrews, só que desta vez, numa amostra de indivíduos leucodermas, brasileiros, com oclusão normal natural, cujo critério seletivo requereu a presença de pelo menos quatro das seis cha- ves de Andrews². Foram observadas quais das seis chaves foram mais prevalentes e quais foram menos freqüentes, bem como a distribuição dos casos de acordo com a quantidade de chaves apresentadas.

\section{MATERIAL E MÉTODO}

A amostra de oclusão normal (Fig. 1) utilizada para a avaliação da prevalência das "Seis chaves de oclusão ótima de Andrews" na população brasileira constituiu-se de 61 pacientes leucodermas, com oclusão normal natural selecionada de acordo com os princípios de Andrews e cuja documentação faz parte do acervo do curso de pós-graduação em Ortodontia da Faculdade de Odontologia da UMESP.

Para a obtenção da amostra utilizada foram examinados 6.118 indivíduos procedentes de várias escolas de ensino fundamental, médio e superior da região do $\mathrm{ABC}$ paulista. Foram selecionados 61 indivíduos, de ambos os gêneros, sendo 36 do gênero feminino e 25 do gênero masculino, na faixa etária compreendida entre 12 e 21 anos (idade média de 16,08), com dentição permanente e oclusão normal natural. Para determinar a oclusão normal natural, alguns critérios foram definidos, buscando encontrar oclusões que apresentassem aspectos de normalidade tanto estéticos quanto funcionais, conforme descrito a seguir:

a) a oclusão deveria apresentar, no mínimo, quatro das seis chaves de oclusão ótima definidas por Andrews em 1972, sendo que a relação interarcos, a primeira das Chaves de Oclusão de Andrews, deveria estar presente obrigatoriamente em todos os casos;
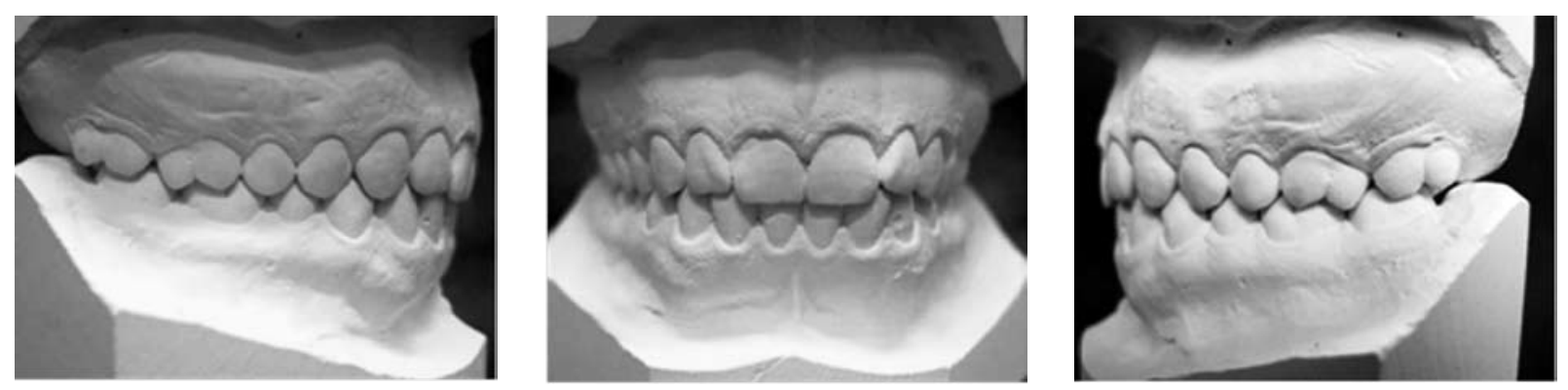

FIGURA 1 - Foto de perfil direito, frente e esquerdo de modelo pertencente à amostra de oclusão normal da UMESP. 
b) a oclusão foi analisada funcionalmente, verificando-se os movimentos mandibulares em protrusiva e em lateroprotrusiva direita e esquerda, não devendo apresentar interferências oclusais em nenhum destes movimentos; e

c) sob manipulação, o fechamento da mandíbula deveria apresentar contatos posteriores simultâneos bilaterais, inclusive nos caninos.

Não deveria haver diferença entre a posição de máxima intercuspidação (MIH) e a posição de relação cêntrica (RC).

Cada uma das seis chaves de oclusão foi analisada conforme a metodologia preconizada por Andrews, na primeira edição traduzida do seu livro Straight Wire - O conceito e o Aparelho, de 1971 ${ }^{1}$.

1) $1^{\text {a }}$ Chave de oclusão: a avaliação da relação molar, pré-molares, caninos e linha média foi feita visualmente e seguiu os sete parâmetros propostos por Andrews (Fig. 2):

1.1) a cúspide mesio-vestibular do primeiro molar superior permanente ocluindo no sulco entre as cúpides vestibulares mesial e mediana do primeiro molar inferior permanente;

1.2) a crista marginal distal do primeiro molar superior ocluindo na crista marginal mesial do segundo molar inferior;

1.3) a cúspide mésio-lingual do primeiro molar superior ocluindo na fossa central do primeiro molar inferior;

1.4) as cúspides vestibulares dos pré-molares superiores possuindo relação de cúspide-ameia com os pré-molares inferiores;

1.5) as cúspides linguais dos pré-molares superiores possuindo uma relação cúspide-fossa com os pré-molares inferiores;

1.6) o canino superior com uma relação cúspideameia com o canino e primeiro pré-molar inferior. A ponta da cúspide levemente mesial à ameia;

1.7) os incisivos superiores sobrepondo-se aos inferiores e as linhas medianas dos arcos coincidentes.

$\mathrm{Na}$ verificação da presença ou ausência desta primeira chave de oclusão, como são avaliados sete fatores, estabelecemos que, para considerar presen- te a primeira chave nos modelos avaliados, deveriam ser observados pelo menos 6 dos 7 fatores.

2) $2^{\text {a }}$ Chave de oclusão: angulações dentárias (Fig. 3)

Para avaliar as angulações dentárias, o longo eixo das coroas dentárias foi demarcado com
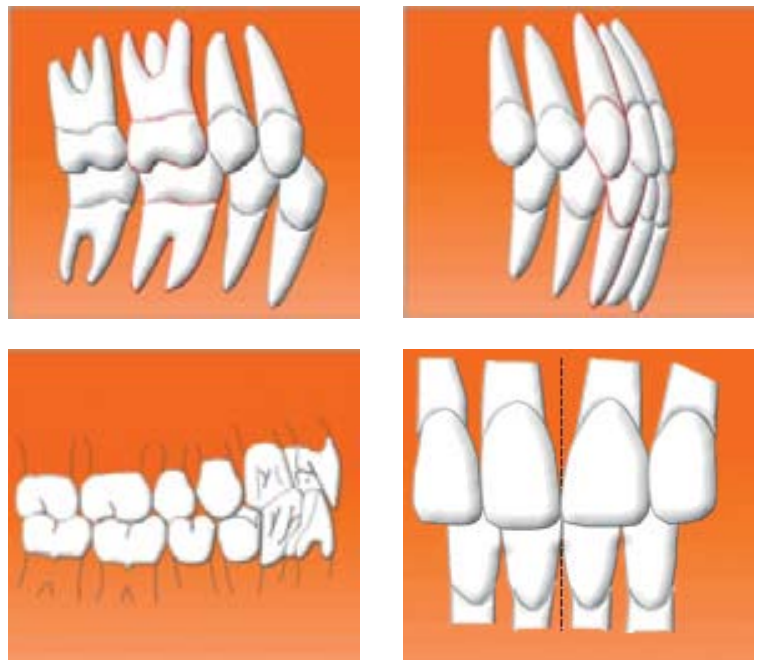

FIGURA 2 - Aferição da $1^{\mathrm{a}}$ chave de oclusão.

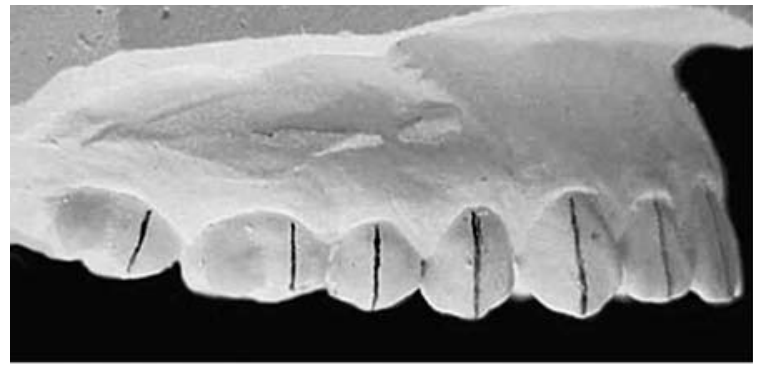

FIGURA 3 - Demarcação dos longos eixos das coroas dentárias para observação das angulações.

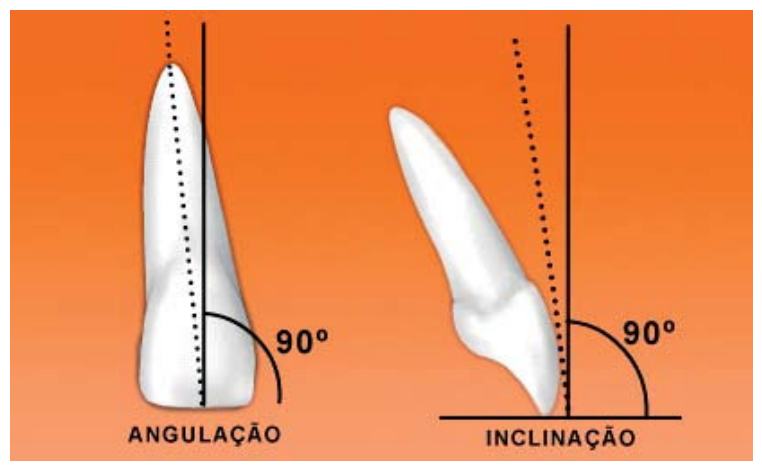

FIGURA 4 - Aferição das inclinações e angulações dentárias. 


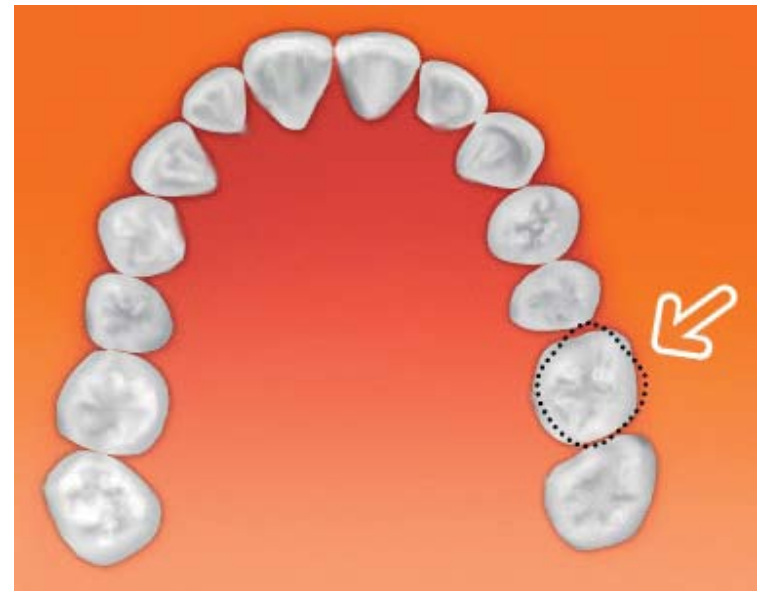

FIGURA 5 - Inspeção da presença de rotações.

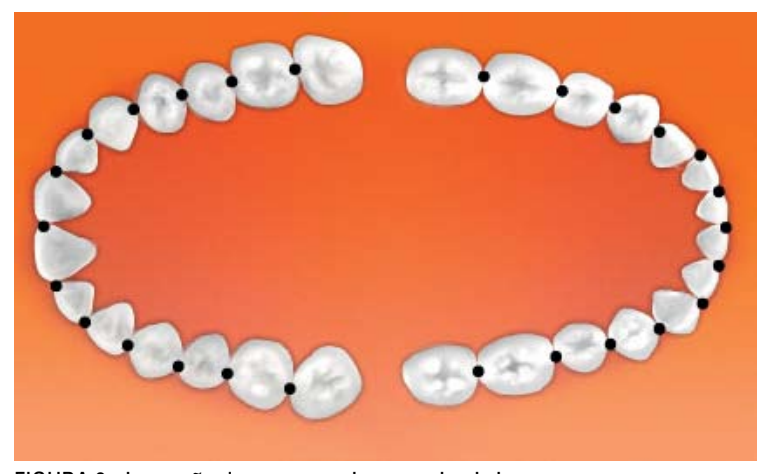

FIGURA 6 - Inspeção dos contatos interproximais justos.

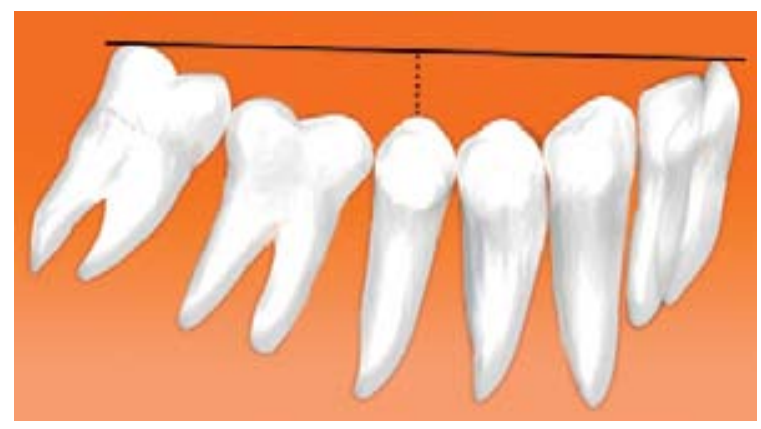

FIGURA 7 - Aferição da curva de Spee.

uma lapiseira de $0,5 \mathrm{~mm}$. Observou-se o posicionamento da porção cervical do longo eixo em relação à porção incisal/oclusal. Conforme Andrews ${ }^{2}$ definiu, quando a porção cervical encontra-se por distal em relação à incisal/oclusal, a angulação é positiva, se por mesial, a angulação é considerada negativa.
3) $3^{\text {a }}$ Chave de oclusão: inclinações dentárias (Fig. 4)

As inclinações dentárias também foram avaliadas utilizando a demarcação dos longos eixos das coroas dentárias. Neste caso, observou-se o posicionamento vestíbulo-lingual das porções cervical e incisal/oclusal. Se a porção incisal/oclusal estivesse vestibularizada em relação à cervical, a inclinação era positiva, se lingualizada, negativa.

4) $4^{\text {a }}$ Chave de oclusão: ausência de rotações (Fig. 5)

A presença de rotações foi verificada por inspeção visual.

5) $5^{\text {a }}$ Chave de oclusão: Contatos interproximais justos (Fig. 6)

A verificação da presença de contatos interproximais também foi realizada por inspeção visual.

6) $6^{a}$ Chave de oclusão: curva de Spee (Fig. 7)

Para visualizar a profundidade da curva de Spee, utilizamos um template transparente, tocando a incisal dos incisivos e a cúspide mais alta do último molar em oclusão, recortado na altura dos caninos para não interferir na aferição. Com uma régua milimetrada, foi mensurada a maior distância entre o template e o pré-molar mais baixo, geralmente o segundo.

\section{RESULTADOS}

Os resultados foram distribuídos em gráficos, demonstrando a prevalência das seis chaves de oclusão na amostra estudada e a distribuição dos casos de acordo com a quantidade de chaves apresentadas (Gráf. 1, 2). Como a $1^{\text {a }}$ chave é constituída por sete fatores complementares da relação interarcos, a prevalência de cada um destes fatores também foi destacada (Gráf. 3, 4).

\section{DISCUSSÃO}

Um paradigma comum em biologia é aquele de que o normal é sempre uma média, no entanto, 


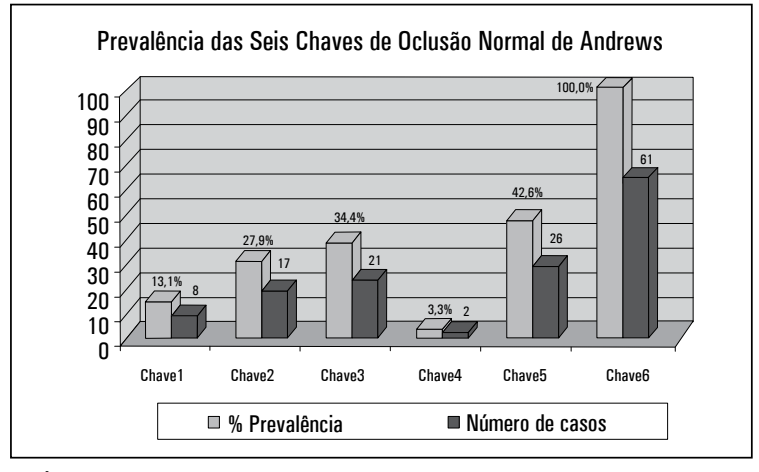

GRÁFICO 1 - Prevalência das seis chaves de oclusão.

mesmo na amostra de oclusão ótima natural de Andrews ${ }^{1}$ havia diferenças, tanto nas relações interarcos quanto nas posições dentárias. Como bem lembrou Andrews², a maior parte da população é atingida pela má oclusão e esse fato poderia até ser interpretado como se os desvios oclusais fossem características inerentes ao ser humano. No entanto, isso não significa que a oclusão esteja confortável, que promova uma mastigação eficiente ou que tenha um aspecto estético saudável, tanto do ponto de vista fisiológico como psicológico.

Quando, em 1899, Angle 3 postulou que em uma oclusão normal a cúspide mésio-vestibular do primeiro molar superior deveria ocluir no sulco vestibular entre a cúspide mésio-vestibular e mediana do primeiro molar inferior, ele não especificou que este fator isolado não seria suficiente para produzir uma oclusão apropriada. Sendo assim, a oclusão pode apresentar-se inadequada, mesmo estando presente a relação molar de Classe I de Angle. Neste estudo, nos propusemos a verificar a prevalência das seis chaves em modelos previamente selecionados de acordo com o método de Andrews $^{2}$, com o intuito de identificar quais as chaves mais e menos prevalentes e quantos indivíduos possuem oclusões preenchendo todos os preceitos oclusais propostos por Andrews.

De acordo com os resultados deste trabalho, todos os modelos avaliados exibiram uma curva de Spee com profundidade menor que $2,5 \mathrm{~mm}$ demonstrando que a $6^{\text {a }}$ chave de oclusão foi a de

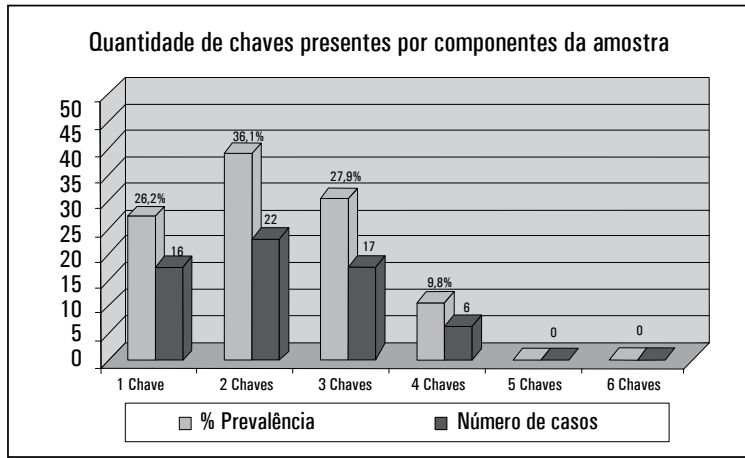

GRÁFICO 2 - Distribuição dos indivíduos de acordo com a quantidade de chaves apresentadas.

maior prevalência (100\%) nesta amostra. Em contrapartida, a $4^{\text {a }}$ chave de oclusão foi a que apresentou menor prevalência $(3,3 \%)$, visto que apenas dois modelos da amostra apresentaram-se completamente livres de rotações. No trabalho anterior, Brangeli ${ }^{5}$ encontrou uma prevalência de $74 \%$ para a $6^{a}$ chave, também a mais prevalente do estudo, sendo a menos prevalente a $5^{\mathrm{a}}$ chave, pontos de contato justos, presente em apenas 10\% daquela amostra. Um outro aspecto observado foi que, apesar das seis chaves serem tidas como elementos interdependentes, a presença das rotações não eliminou necessariamente os pontos de contato entre os dentes, mas provocou modificações na localização e extensão destes pontos, assim como a ausência de contatos justos não necessariamente provocou rotações ${ }^{5}$.

Interessante notar que as inclinações dentárias descritas na $3^{\text {a }}$ chave de oclusão apresentaram uma prevalência de apenas $34,4 \%$, tendo sido observados muitos modelos com inclinação positiva dos incisivos inferiores, o que parece ser um reflexo do padrão facial brasileiro mais convexo. No trabalho anterior, esta chave esteve presente em maior número de casos (52\%). Outro dado curioso foi a baixa prevalência das angulações dentárias, que esteve presente em apenas $27,9 \%$ dos modelos da amostra deste estudo e em 26\% da amostra de Brangeli ${ }^{5}$, sendo que a maioria dos casos não apresentou esta chave devido à angulação negativa observada em grande parte dos incisivos laterais inferiores. Isto nos leva a especular se, na tentativa de preenchermos 


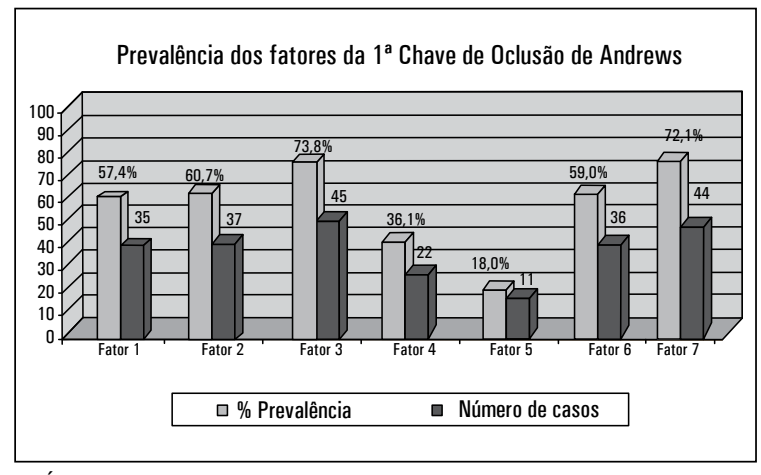

GRÁFICO 3 - Prevalência dos casos apresentando cada um dos sete fatores da primeira chave de oclusão.

os preceitos de Andrews, ao corrigirmos esta angulação negativa dos incisivos inferiores, estaríamos adicionando mais um fator contribuinte para as recidivas na região ântero-inferior.

Vale ressaltar que, de um universo de 6.118 indivíduos, apenas 61 foram selecionados, por apresentarem pelo menos 4 das 6 chaves de Andrews quando examinados clinicamente durante o período de coleta da amostra. Ainda assim, apenas 6 apresentaram efetivamente 4 chaves, sendo que a maioria tinha entre 1 e 3 chaves. Um dos fatores que provavelmente contribuiu para esse resultado, foi o fato de o exame clínico, por si só, não permitir a avaliação completa dos 7 fatores constituintes da $1^{\text {a }}$ chave. Isso pode explicar também o fato de que a $1^{a}$ chave de oclusão foi a segunda menos prevalente na amostra, presente em apenas 8 dos 61 modelos selecionados. No trabalho anterior, cujos modelos foram selecionados de acordo com Angle ${ }^{3}$, dos 97 modelos avaliados, 37 apresentaram esta chave. $\mathrm{E}$, dentre os sete fatores constituintes desta $1^{\text {a }}$ chave, o item que demonstra a relação cúspide-fossa dos pré-molares superiores com os inferiores por lingual, foi o mais dificilmente encontrado. Com base nesta observação, para alcançar as características preconizadas por Andrews ${ }^{1}$, pode ser de grande valia a execução de moldagens periódicas, para visualização da oclusão por lingual nos modelos, com a intenção de acompanhar a evolução do tratamento.

Os dados epidemiológicos, fornecidos por este trabalho, nos convidam a refletir sobre a utilização

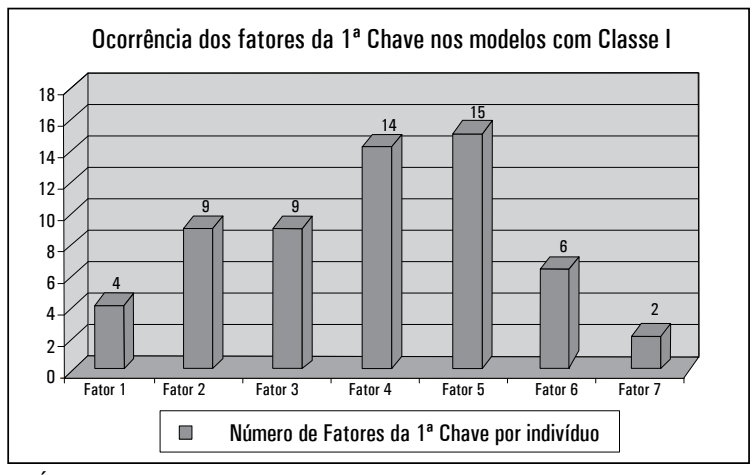

GRÁFICO 4 - Ocorrência dos sete fatores da $1^{\text {a }}$ chave de Andrews nos modelos que apresentaram o $1^{\circ}$ fator da $1^{\text {a }}$ chave (Classe I de Angle).

de conceitos estéticos tão rigorosos para diagnóstico e planejamento de finalização. Pois a prevalência destes apresentou-se extremamente baixa, menos de $1 \%$ da população, considerando a presença de pelo menos 4 chaves, já que não houve na amostra casos em que todas as 6 chaves estivessem presentes, e nem mesmo as 5 chaves, sendo que 16 modelos apresentaram apenas 1 chave.

Para Andrews ${ }^{2}$, é até aceitável não atingirmos todos os objetivos das seis chaves em todos os casos, mas é inadmissível pararmos quando esses são possivelmente alcançáveis. Portanto, as seis chaves para uma oclusão ótima são excelentes parâmetros para diagnóstico e para nos guiarem na busca de qualidade no tratamento de nossos pacientes, no entanto, a ausência de um ou mais destes fatores não deve ser vista rigidamente como insucesso, já que a ocorrência concomitante destas características é muito rara na população. Vale ainda ressaltar que esses objetivos só podem ser esperados em indivíduos com bom relacionamento entre maxila e mandíbula, pois naqueles indivíduos com discrepância esquelética, que serão tratados compensatoriamente, os parâmetros de normalidade vão ser alterados, guiados no sentido de buscar a melhor estética e função possíveis para o caso. Parece-nos claro que a funcionalidade estabelecida na relação interoclusal é o fator de maior importância para a oclusão, independentemente de quais dentes estejam estabelecendo esta relação, desde que possibilite a distribuição equilibrada de forças durante a 
mastigação e excursões mandibulares. Apesar das características oclusais descritas por Andrews², no trabalho das seis chaves, não representarem a média da população, é imperativo considerar a enorme contribuição que trouxeram para a Ortodontia, não apenas por culminarem em outros trabalhos que o levaram a desenvolver o aparelho Straight Wire, mas também por criarem objetivos claros e de melhor qualidade para a finalização dos tratamentos ortodônticos levando, conseqüentemente, à redução da subjetividade dos casos.

\section{CONCLUSÕES}

A avaliação da prevalência das seis chaves de oclusão ótima da Andrews ${ }^{2}$ nesta amostra de oclusão normal, nos permitiu concluir que:

1) dentre os 61 modelos avaliados, apenas 35
$(57,4 \%)$ apresentaram a relação molar de Angle, que é o primeiro fator da $1^{\text {a }}$ chave de Andrews;

2) a maioria dos casos apresentou apenas uma $(26,2 \%)$, duas $(36,1 \%)$ ou três $(27,9 \%)$ chaves de oclusão;

3) a $6^{\text {a }}$ chave de oclusão, curva de Spee suave, foi a de maior prevalência (100\%) na amostra estudada;

4) a $5^{\text {a }}$ chave de oclusão, contatos interproximais justos, foi a de segunda maior prevalência $(42,6 \%)$;

5) nenhum modelo desta amostra apresentou todas as seis chaves de oclusão de Andrews.

\title{
Evaluation of the prevalence of Andrews's six keys to normal occlusion in untreated young brazilian normal occlusion
}

\begin{abstract}
Aim: due to the large use of Andrews's six keys to normal occlusion concept as a tool for diagnosis and treatment planning, we carried out this study with the purpose to evaluate the prevalence of such characteristics in a brazilian sample. Methods: 61 cast models of untreated subjects with normal occlusion were evaluated. The frequency that the six keys were found in each subject, as well as which were the most or least frequent keys, was observed. The results showed that most of the subjects presented one to three keys and none presented all the six keys. Results and Conclusions: the characteristics more frequently observed were: flat curve of Spee (100\%), tight interproximal contacts $(42,6 \%)$ and correct tippings (34.4\%). The low prevalence of untreated normal occlusion that achieves Andrews's requirements lead us to reflect on the search for such rigid patterns.
\end{abstract}

Key words: Orthodontics. Dental occlusion. Normal occlusion.

\section{REFERÊNCIAS}

1. ANDREWS, L. F. Straight wire: o conceito e o aparelho. Califórnia: L. A. Wells, 1971.

2. ANDREWS, L. F. The six keys to normal occlusion. Am J Orthod Dentofacial Orthop, St. Louis, v. 62, no. 3, p. 296-309, Sept. 1972.

3. ANGLE, E. H. Classification of malocclusion. Dent Cosmos, Philadelphia, v. 41, p. 248-357, 1899.

4. ANGLE, E. H. Treatment of malocclusion of the teeth. 7. ed. Philadelphia: S.S. White, 1907.

5. BRANGELI, L. A. M. Prevalência das seis chaves de oclusão de Andrews em jovens brasileiros com oclusão normal. Rev Assoc Paul Cir Dent, São Paulo, v. 55, n. 6, nov./dez. 2001.

6. BROADBENT, B. H. A new $x$-ray technique and its application to orthodontics. Angle Orthod, Appleton, v. 1, no. 2, p. 45-66, Apr. 1931
7. WEINBERGER, C. Orthodontics: an historical review. St. Louis: C. V. Mosby, 1926

8. MOYERS, R. E. et al. Differential diagnosis of class II malocclusions. Part 1. Am J Orthod, St. Louis, v. 78, no. 5, p. 477-494, 1980.

9. WOODSIDE, D. G. Cephalometric roentgenography. In: CLARK J. W. Clinical dentistry. New York: Harper and How, 1980. 